\title{
Dose Reconstruction
}

\section{Q: What is dose reconstruction?}

A: Dose reconstruction is an accepted scientific method used to estimate how much radiation an energy employee was exposed to while working at Department of Energy or Atomic Weapons Employer work sites.

\section{Q: Why is NIOSH doing a dose reconstruction for my claim?}

A: NIOSH is responsible for providing an estimate of the energy employee's radiation dose for the Department of Labor (DOL). DOL will use the final dose reconstruction report to make a recommended decision on your claim.

\section{Q: What information will NIOSH use for my dose reconstruction?}

A: NIOSH may use: film badge readings, medical $x$-rays, urine analysis, incident reports, co-worker data, environmental exposure records, technical documents, interview information, workplace history, and other available information.

Q: How long will it take NIOSH to finish my dose reconstruction?

A: There are many steps necessary to complete each dose reconstruction, and each step varies for each claim. For example, obtaining the information to complete the dose reconstruction estimate may take a significant amount of time depending on the complexity and availability of the data. Therefore, there is no way to predict how long each claim may take to complete the dose reconstruction process.

Q: What if NIOSH cannot do a dose reconstruction?

A: NIOSH will notify you in writing that a dose reconstruction cannot be completed for your claim. NIOSH will provide you with information about a different means of compensation that does not require a dose reconstruction called the Special Exposure Cohort. Further information is available on the NIOSH Web site: www.cdc.gov/niosh/ocas. 
For more information on dose reconstruction, visit www.cdc.gov/niosh/ocas

E-mail questions to: dcas@cdc.gov

or call 1-877-222-7570

DHHS (NIOSH) Publication No. 2005-144

Department of Health and Human Services

Centers for Disease Control and Prevention

National Institute for Occupational Safety and Health 